\title{
International Symposium on Peripheral Neuropathies
}

\section{Place and Date}

The Symposium will be held in Milan (Italy) from Monday, June 26 to Wednesday, June 28, 1978, at the University of Milan (Aula Magna), Via Festa del Perdono 7.

\section{Themes of the Symposium}

The Symposium will mostly deal with metabilic and genetic peripheral neuropathies.

The following fields of interest are tentatively proposed:

- Axonal transport

- Diabetic neuropathies

- Entrapment neuropathies

- Peripheral neuropathies in chronic renal failure.

Contributions on other subjects will be also wellcome: inflammatory-paraneoplastic-endocrine neuropathies; alcoholic neuropathies; abiotrophic and hereditary neuropathies; neuropathies associated with collagen diseases and toxic conditions.

\section{Official Language}

The official language will be English.

\section{Scientific Programm}

The Symposium will include lectures presented by invited speakers (lasting 30 minutes) and free communication (limited to $10^{\prime}$ minutes presentation and 5 minutes' discussion).

\section{Presentations of Abstracts}

A special form for submitting abstracts with detailed instructions will be sent to those who are interested (see the tear-off portion of this announcement).

Abstracts should be forwarded for consideration by the Organizing Committee not later than 15 March 1978.

It is inderstood that Authors will register in due time as an active partecipant to the Symposium.

Abstracts will be published in the abstract volume for circulation during the Symposium.

\section{Enquiries}

All enquiries about the Symposium should be addressed to:

Secretariat - Symposium on "Peripheral Neuropathies", Ospedale S. Raffaele, Tel. 02-2134041 I-20090 Segrate - Milan, Italy 